University of Nebraska - Lincoln

DigitalCommons@University of Nebraska - Lincoln

Timothy J. Gay Publications

Research Papers in Physics and Astronomy

December 1982

\title{
Measurement of spin-exchange effects in electronhydrogen collisions: Further studies of impact ionization
}

Timothy J. Gay

University of Nebraska - Lincoln, tgay1@unl.edu

G. D. Fletcher

Yale University, New Haven, Connecticut

M. J. Alguard

Yale University, New Haven, Connecticut

V. W. Hughes

Yale University, New Haven, Connecticut

P. F. Wainwright

Yale University, New Haven, Connecticut

See next page for additional authors

Follow this and additional works at: https://digitalcommons.unl.edu/physicsgay

Part of the Physics Commons

Gay, Timothy J. ; Fletcher, G. D.; Alguard, M. J.; Hughes, V. W.; Wainwright, P. F.; and Lubell, M. S., "Measurement of spin-exchange effects in electronhydrogen collisions: Further studies of impact ionization" (1982). Timothy J. Gay Publications. 10.

https://digitalcommons.unl.edu/physicsgay/10

This Article is brought to you for free and open access by the Research Papers in Physics and Astronomy at DigitalCommons@University of Nebraska - Lincoln. It has been accepted for inclusion in Timothy J. Gay Publications by an authorized administrator of DigitalCommons@University of Nebraska - Lincoln. 


\section{Authors}

Timothy J. Gay, G. D. Fletcher, M. J. Alguard, V. W. Hughes, P. F. Wainwright, and M. S. Lubell 
Phys. Rev. A 26, 3664 - 3667 (1982)

[Issue 6 - December 1982]

\title{
Measurement of spin-exchange effects in electron- hydrogen collisions: Further studies of impact ionization
}

\author{
T. J. Gay, G. D. Fletcher, M. J. Alguard *, V. W. Hughes, and P. F. Wainwright ${ }^{\dagger}$ \\ J. W. Gibbs Laboratory, Yale University, New Haven, Connecticut 06520
}

M. S. Lubell

J. W. Gibbs Laboratory, Yale University, New Haven, Connecticut 06520 and Physics Department, The City College of the City University of New York, New York, New York 10031

Received 14 July 1982

Using a Fano-effect polarized electron source and a state-selected thermally dissociated hydrogen beam, we measured the interference between the direct and exchange scattering amplitudes for electron-impact ionization of atomic hydrogen between 14.1 and $30.3 \mathrm{eV}$. We report the data from these measurements and the results of corrections applied to previously published data.

C1982 The American Physical Society

URL: http://link.aps.org/abstract/PRA/v26/p3664

DOI: 10.1103/PhysRevA.26.3664 


\title{
Measurement of spin-exchange effects in electron-hydrogen collisions: Further studies of impact ionization
}

\author{
T. J. Gay, G. D. Fletcher, M. J. Alguard, ${ }^{*}$ V. W. Hughes, and P. F. Wainwright ${ }^{\dagger}$ \\ J. W. Gibbs Laboratory, Yale University, New Haven, Connecticut 06520 \\ M. S. Lubell \\ J. W. Gibbs Laboratory, Yale University, New Haven, Connecticut 06520 \\ and Physics Department, The City College of the City University of New York, \\ Now York, New York 10031 \\ (Received 14 July 1982)
}

\begin{abstract}
Using a Fano-effect polarized electron source and a state-selected thermally dissociated hydrogen beam, we measured the interference between the direct and exchange scattering amplitudes for electron-impact ionization of atomic hydrogen between 14.1 and $30.3 \mathrm{eV}$. We report the data from these measurements and the results of corrections applied to previously published data.
\end{abstract}

The first measurements of spin dependence in electron-impact ionization of atomic hydrogen, reported several years ago, ${ }^{1}$ exhibited significant differences with theoretical predictions over an energy range from threshold to approximately $50 \mathrm{eV}$. Motivated by a desire to augment these measurements and to provide a consistency check for new measurements of spin dependence in $90^{\circ}$ elastic scattering, ${ }^{2}$ we accumulated additional ionization data for incident electron energies from 14.1 to $30.3 \mathrm{eV}$. The apparatus permitted us to obtain the additional ionization data and the elastic scattering data simultaneously.

The quantity which we determined is the asymmetry $A_{I} \equiv\left[\sigma_{I}(\uparrow \downarrow)-\sigma_{I}(\uparrow \uparrow)\right] /\left[\sigma_{I}(\uparrow \downarrow)+\sigma_{I}(\uparrow \uparrow)\right]$, where $\sigma_{I}$ is the total ionization cross section for the spins of the incident and atomic electrons antiparallel $(\uparrow \downarrow)$ or parallel $(\uparrow \uparrow)$. In terms of the direct and exchange amplitudes, $f$ and $g$, respectively, and the spin-averaged total cross section $\bar{\sigma}_{I}$ the asymmetry can be expressed as

$$
A_{I}=\iint \frac{|f||g| \cos \theta d \overrightarrow{\mathrm{k}}_{1}^{\prime} d \overrightarrow{\mathrm{k}}_{2}^{\prime}}{\bar{\sigma}_{I}},
$$

where the integral extends over the allowed momenta $\overrightarrow{\mathrm{k}}_{1}^{\prime}$ and $\overrightarrow{\mathrm{k}}_{2}^{\prime}$ of the two outgoing electrons and $\theta$ is the relative phase between $f$ and $g$. Alternatively, we can write $A_{I}=(1-r) /(1+3 r)$, where $r$ is the ratio of the triplet to singlet total cross sections.

In this report, we present our new ionization measurements, compare them with the older data, and discuss several corrections which we have applied to the older data. A complete description of the data analysis will appear in a future publication.

The experimental method and apparatus have been described previously. ${ }^{1-3}$ We include herein the most relevant details. Longitudinally polarized electrons from a Fano-effect ${ }^{3}$ source intersected at right angles a chopped beam of thermally dissociated stateselected hydrogen atoms whose polarization vector was oriented either antiparallel or parallel to that of the incident electrons in accordance with the direction of a $\sim 100-\mathrm{mG}$ magnetic field in the interaction region. Protons produced in $e^{-}-\mathrm{H}$ collisions were deflected out of the primary beam downstream from the interaction region and were detected by an electron multiplier. Further downstream, a quadrupole mass analyzer (QMA) monitored the relative amounts of $\mathrm{H}$ and $\mathrm{H}_{2}$ in the undeflected neutral beam.

We accumulated data in a series of runs during each of which the electron beam polarization was reversed frequently by $90^{\circ}$ rotation of a quarter-wave plate in the optical train of the Fano source. ${ }^{3}$ For each of these runs, we defined a "real" asymmetry $\Delta_{R}$ as

$$
\Delta_{R}=\frac{ \pm\left(N_{02}-N_{13}-B_{02}+B_{13}\right)}{N_{02}+N_{13}-B_{02}-B_{13}},
$$

where $N_{02}$ is the sum of H-beam-on ion counts for quarter-wave-plate positions 0 and $2\left(0^{\circ}\right.$ and $\left.180^{\circ}\right)$, $N_{13}$ is the equivalent sum for quarter-wave-plate positions 1 and $3\left(90^{\circ}\right.$ and $\left.270^{\circ}\right)$, and $B_{02}$ and $B_{13}$ are the corresponding $\mathrm{H}$-beam-off sums. The positive (negative) sign in Eq. (2) applies when the H-beam polarization is oriented such that $N_{02}$ corresponds to electron and $\mathrm{H}$ spins antiparallel (parallel). The experimental asymmetry $\Delta_{R}$ is related to $A_{I}$ by $\Delta_{R}$ $=P_{\mathrm{H}} P_{e}\left(1-F_{2}\right)|\cos \alpha| A_{I}$, where $P_{\mathrm{H}}$ is the hydrogen polarization $(0.50 \pm 0.02), P_{e}$ is the electron polarization $(0.61$ to $0.75 \pm 0.04), \alpha\left(\leq 10^{\circ}\right)$ is the angle between the $\mathrm{H}$ polarization vector and electron beam, 
and $F_{2}$ is the fraction of counts resulting from ionization of $\mathrm{H}_{2}(5-20 \%)$.

In order to check for systematic effects associated with reversal of the electron beam polarization, we constructed, in addition to the real asymmetry, two false asymmetries, $\Delta_{F 1}$ and $\Delta_{F 2}$, corresponding to quarter-wave-plate combinations $(0)+(1)-(2)-(3)$ and $(0)+(3)-(1)-(2)$, respectively. Within statistical uncertainty and in the absence of systematic effects, these false asymmetries should be zero.

As in previous studies, ${ }^{1,3}$ we determined the electron polarization by $\sim 100-\mathrm{keV} 120^{\circ}$ Mott scattering from Formvar-backed gold foil targets with the use of two Si surface-barrier electron detectors. We define the Mott asymmetry $\Delta_{M}$ as $\Delta_{M}=(1-\xi) /(1+\xi)$, where $\xi=\left(N_{1}^{+} N_{2}^{-} / N_{1}^{-} N_{2}^{+}\right)^{1 / 2}$ and $N$ is the number of counts from detectors 1 and 2 for positive or negative helicity of the polarized electrons emerging from the Fano source. Values of $N$ include corrections for detector and electronic noise, elastic and inelastic scattering from the Formvar backing, inelastic scattering from the gold target and the chamber walls, and backscattering from the detectors. With $\Delta_{M}$ determined for several target thicknesses, we calculated $P_{e}$ from the expression $P_{e}=\Delta_{M}(0) / S$, where $\Delta_{M}(0)$ is the Mott asymmetry extrapolated to zero target thickness and $S$, the Sherman function for our particular experimental arrangement, is taken to be $0.387 \pm 0.008$.

In order to determine the molecular fraction $F_{2}$ we compared measurements made at the "hot" operating temperature $(\sim 2800 \mathrm{~K})$ of the hydrogen oven

TABLE I. Results of data analysis (corrected data of Ref. 1 in italics).

\begin{tabular}{|c|c|c|c|c|c|}
\hline \multirow{2}{*}{$\begin{array}{c}\text { Energy }^{\mathrm{a}} \\
(\mathrm{eV})\end{array}$} & \multirow[b]{2}{*}{$A_{I}$} & \multirow{2}{*}{$\begin{array}{c}\Delta_{F 1}^{\mathrm{b}} \\
\left(\times 10^{-4}\right)\end{array}$} & \multirow{2}{*}{$\begin{array}{c}\Delta_{F 2^{\mathrm{b}}} \\
\left(\times 10^{-4}\right)\end{array}$} & \multicolumn{2}{|c|}{$\chi^{2}(0) /$ deg. freedom } \\
\hline & & & & $\Delta_{F 1}$ & $\Delta_{F 2}$ \\
\hline $14.1(2.3)$ & $0.429\left({ }_{-36}^{+38}\right)$ & $-7(7)$ & $-1(7)$ & $16 / 14$ & $20 / 14$ \\
\hline $15.0(3.2)$ & $0.478\left( \pm_{-98}^{+101}\right)$ & $-117(57)$ & $-16(57)$ & $15 / 13$ & $9 / 13$ \\
\hline $17.0(3.2)$ & $0.434(39)$ & $77(42)$ & $-66(42)$ & $8 / 10$ & $8 / 10$ \\
\hline $17.2(2.3)$ & $0.491\left( \pm_{-37}^{+42}\right)$ & $-7(20)$ & $16(20)$ & $8 / 16$ & $20 / 16$ \\
\hline $19.0(3.2)$ & $0.435(43)$ & $54(36)$ & $98(36)$ & $10 / 10$ & $18 / 10$ \\
\hline $20.1(2.7)^{\mathrm{c}}$ & $0.405(29)$ & $-17(22)$ & $18(22)$ & $5 / 9$ & $15 / 9$ \\
\hline $22.2(2.5)$ & $0.409( \pm 50)$ & $13(8)$ & $7(8)$ & $20 / 19$ & $21 / 19$ \\
\hline $23.0(3.2)$ & $0.428\left( \pm_{-42}^{+41}\right)$ & $-69(35)$ & $-5(35)$ & $16 / 9$ & $11 / 9$ \\
\hline $24.3(2.7)$ & $0.415( \pm 29)$ & $-14(10)$ & $2(10)$ & $12 / 12$ & $9 / 12$ \\
\hline $27.0(2.7)$ & $0.346\left({ }_{-49}^{+53}\right)$ & $17(12)$ & $6(12)$ & $25 / 26$ & $27 / 26$ \\
\hline $27.0(3.2)$ & $0.384\left({ }_{-45}^{+43}\right)$ & $5(7)$ & $-10(7)$ & $78 / 68$ & $78 / 68$ \\
\hline $30.3(2.5)$ & $0.302\left({ }_{-30}^{45}\right)$ & $3(8)$ & $-16(8)$ & $20 / 17$ & $17 / 17$ \\
\hline $34.0(3.2)$ & $0.316\left({ }_{-33}^{+35}\right)$ & $-60(24)$ & $32(24)$ & $38 / 6$ & $9 / 6$ \\
\hline $42.0(3.2)$ & $0.310(25)$ & $3(17)$ & $35(17)$ & $8 / 10$ & $20 / 10$ \\
\hline $57.0(3.2)$ & $0.236(21)$ & $-6(23)$ & $0(23)$ & $26 / 9$ & $11 / 9$ \\
\hline $77.0(3.2)$ & $0.185\left(\left(_{-23}^{+24}\right)\right.$ & $7(47)$ & $-19(47)$ & $15 / 9$ & $7 / 9$ \\
\hline $107.0(3.2)^{\mathrm{d}}$ & $0.143( \pm-16)$ & $24(17)$ & $-6(17)$ & $25 / 12$ & $7 / 12$ \\
\hline $147.0(3.2)^{\mathrm{d}}$ & $0.118\left({ }_{-16}^{+15}\right)$ & $13(18)$ & $20(18)$ & $11 / 8$ & $7 / 8$ \\
\hline $197.0(3.2)^{\mathrm{d}}$ & $0.071(15)$ & $-26(23)$ & $-3(23)$ & $12 / 8$ & $3 / 8$ \\
\hline All runs & (Ref. 1) & $2(5)$ & $1(5)$ & $261 / 172$ & $189 / 172$ \\
\hline All runs & (This work) & $0(4)$ & $0(4)$ & $107 / 113$ & $130 / 113$ \\
\hline
\end{tabular}

aElectron beam energy spread (full width at half maximum) in parentheses.

${ }^{b}$ As distinct from false asymmetries of Ref. 1 , all values have background $B$ subtacted.

cData for NOR field only.

dData for REV field only. 
with those made at a "cold" temperature $(\sim 1400$ $\mathrm{K})$, where the hydrogen beam is essentially molecular in composition. With $\Lambda$ defined by $\Lambda \equiv(N-B) / Q_{2}$, where $Q_{2}$ is the QMA signal for $\mathrm{H}_{2}$ and $N$ and $B$ are now summed over all four quarter-wave-plate positions, it can be shown that $F_{2}=\Lambda_{\text {cold }} / \Lambda_{\text {hot }}$. For conditions of constant beam geometry and constant QMA and ion detector efficiencies, it can also be shown that $\Lambda=\alpha+\beta \Gamma$, where $\alpha$ and $\beta$ are positive constants, $\Gamma=Q_{1} / Q_{2}$, and $Q_{1}$ is the QMA signal for $\mathrm{H}$.

A typical measurement of $A_{I}$ at one energy consisted of four to eight runs for each of the two orientations of the $\mathrm{H}$-target polarization. For each run we calculated the quantity $\Delta_{R} /\left(1-F_{2}\right)$ and its associated uncertainty and then performed a $\chi^{2}$ analysis first for the two target polarizations individually and then for both groups taken together. If no systematic effects were observed, the individual run results were combined to give a statistically weighted average for $A_{I}$ with the uncertainties of $P_{e}$ and $P_{\mathrm{H}}$ added in quadrature.

At incident energies of 22.2, 27.0, and $30.3 \mathrm{eV}$, however, the values of $A_{I}$ obtained for one magnetic field orientation (designated NOR) were consistently $5-20 \%$ lower than those obtained for the opposite orientation (designated REV). In the earlier work of Ref. 1 a similar effect was observed at incident energies of 15.0 and $27.0 \mathrm{eV}$ and was attributed to uncompensated magnetic field components transverse to the electron beam for the NOR orientation, a conclusion reinforced by a diminution of the effect as the magnitude of the longitudinal field was increased from 100 to $200 \mathrm{mG}$. Based upon the results of the present work, we now believe that this conclusion is erroneous, since the elastic scattering data do not display any such systematic effects. Instead, we believe that the acceptance of the ion detector is slightly field dependent, a result that, in retrospect, is not unreasonable given the geometry and the fields involved. Since there is no longer any reason to assume $a$ priori that the REV data are the correct ones - rather they may simply be reflective of a restricted range of angular acceptance for which the asymmetry is higher - we treated the data equally, instead of correcting all NOR data upward by $6 \%$ as was done in Ref. 1. The nonstatistical spread of the data necessitated a modification of the analysis procedure with a resultant increase in the size of the quoted uncertainties. These procedural modifications were applied to both the present data and those of Ref. 1. Data obtained at $30.3 \mathrm{eV}$ (this work) and $107.0 \mathrm{eV}$ (Ref. 1) displayed some additional nonstatistical behavior whose origin is not well understood. Consequently, we increased the uncertainties at these energies slightly.

The sensitivity of the acceptance of the ion detector to experimental operating conditions also affected the determination of $F_{2}$. In the analysis of the $\Lambda-\Gamma$ data we found that for the present ionization measurements the dependence of $\Lambda$ on $\Gamma$ became nonlinear for hydrogen oven temperatures below $\sim 1600$ $\mathrm{K}$. We did not observe this behavior in the elastic scattering data, nor was it observed in the work of Ref. 1. We believe it to be due to a small change in the detector geometry, made prior to this work, which caused a reduction of the acceptance of the detector for ions produced from cold, slowly moving $\mathrm{H}_{2}$ molecules. Corrections for this low-temperature rolloff increased the values of $A_{I}$ from the present work by $4-5 \%$.

The $F_{2}$ determination was also affected by the presence of a background asymmetry $\left(<\Delta_{R} / 4\right)$ in the hot H-beam-off signal, which we traced to the velocity spread of the hydrogen beam and the constraints on the data-acquisition timing gates. This asymmetry introduced an uncertainty in the precision of our measurement of $F_{2}$ which was not taken into account in the results reported in Ref. 1 . We found that the

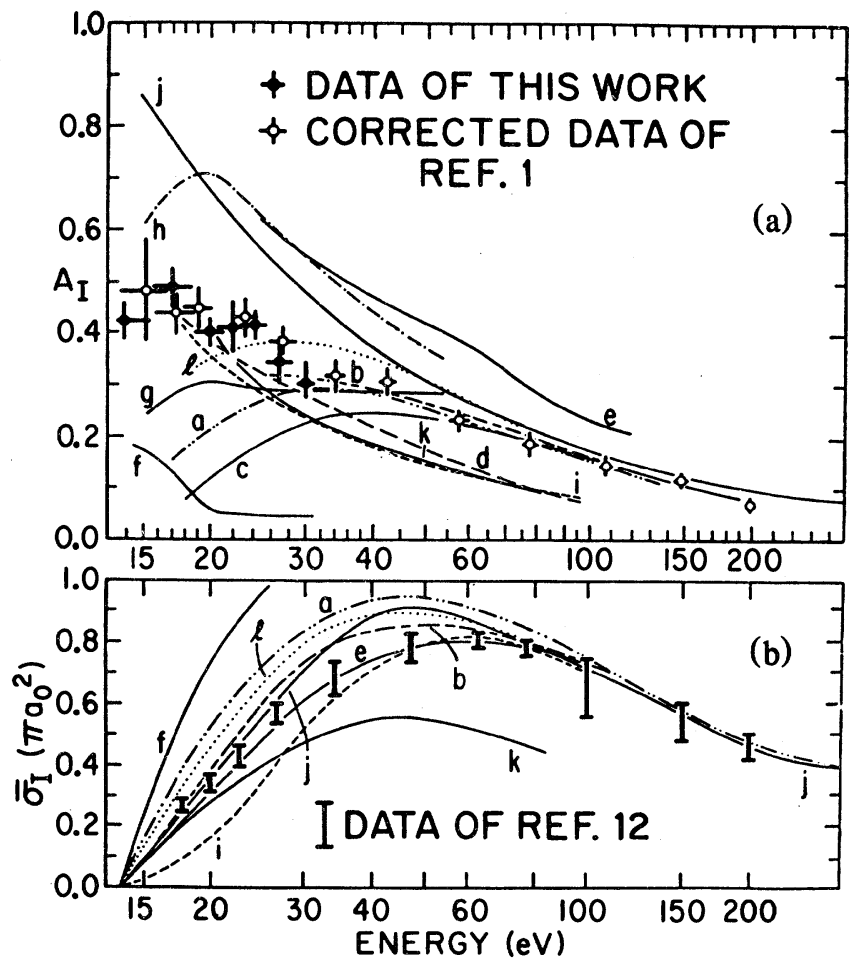

FIG. 1. (a) Measured values of $A_{I}$ as a function of incident electron energy. Vertical error bars include statistical and systematic uncertainties. Horizontal bars indicate full width at half maximum energy spread of the electron beam. The theoretical curves are obtained from information in the following references using the procedure given in Ref. 2: Curves a, g, and h, Ref. 4; b and e, Ref. 5; c, Ref. 6; d and i, Ref. 7; f, Ref. 8; j, Ref. 9; k, Ref. 10; 1, Ref. 11. (b) Experimental values of $\bar{\sigma}$ obtained by other investigators; vertical bars indicate the spread of the measurements. Theoretical curves are from references given above. 
background asymmetry necessitated a $\sim 5 \%$ increase in the $A_{I}$ uncertainties for both the new and old data.

In comparing the present measurements of $A_{I}$ with those of Ref. 1, we discovered a small systematic discrepancy which lay beyond the bounds of statistics. An examination of the corresponding values of $P_{e}$ revealed a discrepancy of approximately the same direction and size, prompting us to compare in detail the Mott scattering analysis used in both cases. We discovered that the data in Ref. 1 had not been corrected properly for elastic scattering from the Formvar backing or for the asymmetry in the inelastic background, which depends on the initial helicity of the electrons. The inclusion of these two corrections caused a 5\% increase in the values of $P_{e}$ and a small increase in their associated uncertainties. With these corrections and those described previously, the values of $A_{I}$ from Ref. 1 decreased by typically $7 \%$ of themselves ( $\sim 1$ standard deviation) and their corresponding errors increased by $\sim 50 \%$.

The results of $A_{I}$ from the present studies and the corrected results from Ref. 1 appear in Table I together with the corresponding false asymmetries $\Delta_{F 1}$ and $\Delta_{F 2}$. While the reduced $\chi^{2}$ values for $\Delta_{F 1}$ and $\Delta_{F 2}$ are nonstatistical in several cases, indicating the presence of some uncorrected systematic effects, the values of $\Delta_{F 1}$ and $\Delta_{F 2}$ themselves are so small that we believe these residual systematic effects have a negligible influence on $A_{I}$.

The numerical results for $A_{I}$ given in Table I appear in graphical form in Fig. 1 (a) along with measurements of $\bar{\sigma}_{I}$ of other workers ${ }^{12}$ [Fig. 1(b)] and a representative sample of theoretical predictions.

From the graphical presentation we can make several observations. First, our new data are in substantial agreement with the corrected data of Ref. 1. Second, with the caveat that $A_{I}$ does not rise abruptly to unity at threshold, both sets of data support the claim of Greene and $\mathrm{Rau}^{13}$ that Klar and Schlecht ${ }^{14}$ were incorrect in predicting that at threshold $A_{I}=1$. Finally, several theories agree reasonably well with the measured values of $\bar{\sigma}_{I}$, but there is substantial disagreement between our results and all of the available theoretical predictions between threshold and $\sim 50$ $\mathrm{eV}$, thus suggesting that polarization experiments provide a sensitive test of theoretical approximation methods for the difficult problem of impact ionization.

We thank W. Raith, C. W. Tu, F. C. Tang, and R. Broughton for their contributions to this work. One of us (M.S.L.) wishes to thank the Sloan Foundation for support in the form of an Alfred P. Sloan Fellowship. This work was supported in part by the National Science Foundation (Yale Grants No. PHY8105035 and No. PHY81-08680) and the City University of New York (Grant No. PSC-CUNY RF 13750).
"Permanent address: Measurex Corporation, Cupertino, Calif. 95014.

${ }^{\dagger}$ Permanent address: Bell Laboratories, Holmdel, N. J. 07733.

${ }^{1}$ M. J. Alguard et al., Phys. Rev. Lett. 39, 334 (1977); M. S. Lubell, in Coherence and Correlation in Atomic Collisions, edited by H. Kleinpoppen and J. F. Williams (Plenum, New York, 1980), p. 663.

${ }^{2}$ G. D. Fletcher et al., Phys. Rev. Lett. 48, 1671 (1982).

3P. F. Wainwright et al., Rev. Sci. Instrum. 49, 571 (1978).

${ }^{4}$ M. R. H. Rudge and M. J. Seaton, Proc. R. Soc. London, Ser. A 283,262 (1965).

${ }^{5}$ R. J. Peterkop, Zh. Eksp. Teor. Fiz. 41, 1938 (1961) [Sov. Phys. JETP 14, 1377 (1962)].

6S. Geltman et al., Proc. Phys. Soc. London 81, 375 (1963).
${ }^{7}$ J. E. Goldin and J. H. McGuire, Phys. Rev. Lett. $\underline{32}, 1218$ (1974).

${ }^{8}$ M. R. H. Rudge and S. B. Schwartz, Proc. Phys. Soc. London 88,563 (1966).

${ }^{9}$ V. I. Ochkur, Zh. Eksp. Teor. Fiz. 47, 1746 (1964) [Sov. Phys. JETP 20, 1175 (1964)].

${ }^{10}$ D. F. Gallaher, J. Phys. B 7, 362 (1974).

${ }^{11}$ M. R. H. Rudge, J. Phys. B 11 , L149 (1978).

${ }^{12}$ W. L. Fite and R. T. Brackman, Phys. Rev. 112, 1141 (1958), E. W. Rothe et al., ibid. $\underline{125}, 582(1962)$ and references therein.

${ }^{13}$ C. H. Greene and A. R. P. Rau, Phys. Rev. Lett. $\underline{48}, 533$ (1982).

${ }^{14}$ H. Klar and W. Schlecht, J. Phys. B 9 , 1699 (1976). 